\title{
Effect of an Additional, Parallel Capacitor on Pulsed Inductive Plasma Accelerator Performance
}

\author{
IEPC-2011-160 \\ Presented at the $32^{\text {nd }}$ International Electric Propulsion Conference, Wiesbaden, Germany \\ September 11-15, 2011 \\ Kurt A. Polzin* Amy D. Sivak ${ }^{\dagger}$ and Joseph V. Balla \\ NASA-Marshall Space Flight Center, Huntsville, AL 35812
}

\begin{abstract}
A model of pulsed inductive plasma thrusters consisting of a set of coupled circuit equations and a one-dimensional momentum equation has been used to study the effects of adding a second, parallel capacitor into the system. The equations were nondimensionalized, permitting the recovery of several already-known scaling parameters and leading to the identification of a parameter that is unique to the particular topology studied. The current rise rate through the inductive acceleration coil was used as a proxy measurement of the effectiveness of inductive propellant ionization since higher rise rates produce stronger, potentially better ionizing electric fields at the coil face. Contour plots representing thruster performance (exhaust velocity and efficiency) and current rise rate in the coil were generated numerically as a function of the scaling parameters. The analysis reveals that when the value of the second capcitor is much less than the first capacitor, the performance of the two-capacitor system approaches that of the single-capacitor system. In addition, as the second capacitor is decreased in value the current rise rate can grow to be twice as great as the rise rate attained in the single capacitor case.
\end{abstract}

\section{Introduction}

$\mathrm{P}$ ULSED inductive plasma accelerators are electrodeless space propulsion devices where a capacitor is charged to an initial voltage and then discharged as a high-current pulse through a coil, inductively coupling energy into the propellant. The field produced by this pulse ionizes the propellant, producing a plasma near the face of the coil. Once a plasma is formed it can be accelerated and expelled at a high exhaust velocity by the Lorentz force arising from the interaction of an induced plasma current and the magnetic field. While there are many coil geometries that can be employed to inductively accelerate a plasma, in this paper the discussion is limited to planar geometries where the coil takes the shape of a flat spiral. A recent review of the developmental history of planar-geometry pulsed inductive thrusters can be found in Ref. [1]. Two concepts that have employed this geometry are the Pulsed Inductive Thruster (PIT) ${ }^{2,3}$ and the Faraday Accelerator with Radio-frequency Assisted Discharge (FARAD). ${ }^{4}$

Pulsed inductive plasma accelerators possess many demonstrated and potential benefits, ${ }^{1,3}$ providing motivation for continued investigation. The electrodeless nature of these thrusters eliminates electrode erosion, mitigating the lifetime and contamination issues associated with conventional electric thrusters. Also, a wider variety of propellants is available for use when compatibility with metallic electrodes is no longer an issue. Pulsed inductive accelerators have demonstrated operation on propellants like ammonia, hydrazine, and $\mathrm{CO}_{2}$, and there is no fundamental reason why they would not operate on other propellants like $\mathrm{H}_{2} \mathrm{O}$. It is well known that for a given propellant pulsed accelerators can maintain constant specific impulse $I_{\mathrm{sp}}$ and thrust efficiency $\eta_{\mathrm{t}}$ over a wide range of input power levels by adjusting the pulse rate to maintain a constant discharge energy per pulse. In addition, these thrusters have demonstrated operation

${ }^{*}$ Propulsion Research Engineer, Propulsion Research and Technology Applications Branch, Propulsion Systems Department. kurt.a.polzin@nasa.gov

${ }^{\dagger}$ Presently: Aerospace Engineer, Thrust Vector Control Systems Integration and Components Branch, Propulsion Systems Department.

${ }_{\ddagger}^{\ddagger}$ Presently: Graduate Research Assistant, The Ohio State University, Columbus, OH. 
A)

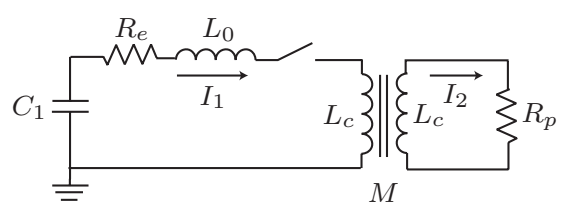

B)

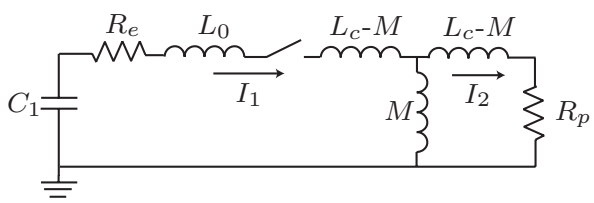

C)

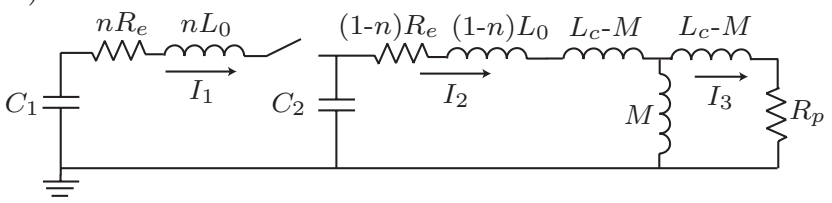

Figure 1. A) General lumped-element circuit model and B) equivalent circuit model of a pulsed inductive accelerator (after Ref. [2]). C) Equivalent electrical circuit model of an accelerator with a second, parallel capacitor.

in a regime where $\eta_{\mathrm{t}}$ is relatively constant over a wide range of $I_{\mathrm{sp}}$. Finally, thrusters in this class have operated at high energy per pulse, and by increasing the pulse rate they offer the potential to process very high levels of power to provide relatively high thrust using a single thruster.

Pulse circuits for inductive thrusters have in the past typically been limited to a simple, ringing resistiveinductive-capacitive (RLC) configuration like that shown in Fig. 1A and redrawn in Fig. 1B as an equivalent circuit that is easier to model. However, as the field has developed proposed circuit topologies are becoming more complex. ${ }^{5}$ In this paper, we proceed with an investigation of the circuit shown in Fig. $1 \mathrm{C}$ where a second capacitor with value less than or equal to $C_{1}$ is inserted downstream of the switch. This study was motivated by a data set where the efficiency of a pulsed inductive thruster increased when the capacitor $C_{2}$ was added. ${ }^{6}$ Unfortunately, the value of $C_{1}$ was also increased when $C_{2}$ was added and previous work has shown that increasing $C_{1}$ can also increase the efficiency. ${ }^{7}$ The authors of Ref. [6] additionally noted that the voltage across $C_{2}$ could be approximately double the charge voltage across $C_{1}$ when $C_{2} \ll C_{1}$. This result was interesting because it implied that the voltage and commensurate current rise rate in the coil could be increased by adding $C_{2}$. A higher current rise rate can, in turn, produce stronger electric fields at the coil face and potentially lead to better inductive ionization of the propellant. ${ }^{5}$

There exists a 1-D pulsed inductive acceleration model that employs a set of circuit equations coupled to a one-dimensional momentum equation..$^{2,3}$ The model has since been nondimensionalized and used by Polzin et al..$^{7,8}$ to define a set of scaling parameters and gain general insight into their effects on thruster performance. In this paper we modify the acceleration model to account for the presence of $C_{2}$ in the system and then nondimensionalize the equation set to identify any new nondimensional scaling parameters that arise for the new circuit topology. The current rise rate through the coil is computed for various cases, and it is used as a proxy measurement to gauge the ability of the coil to inductively ionize the propellant. Finally, we examine whether the addition of $C_{2}$ imposes potential benefits or detriments on thruster efficiency and $I_{\mathrm{sp}}$.

\section{Governing Equations}

\section{A. Physical Model}

A circuit-based model of a pulsed inductive accelerator previously developed by Lovberg and Dailey ${ }^{2}$ is modified to account for the addition of the second capacitor as shown in Fig. 1C. In the lumped-element circuit model shown in Fig. 1A,B, the external circuit (left side of the figure) possesses a capacitor bank with capacitance $C_{1}$, external inductance $L_{0}$, resistance $R_{e}$, and acceleration coil inductance $L_{C}$. The plasma also has an inductance equal to $L_{C}$ and a resistance $R_{p}$. The two circuits are inductively coupled through 
the coil, which acts as a transformer with mutual inductance $M$. The value of $M$ is a function of the current sheet position $z$. In Fig. $1 \mathrm{C}$, a second capacitor $C_{2}$ is added between the primary capacitor bank and the inductive coil. This has the effect of splitting the $L_{0}$ and $R_{e}$, with the value on the left and right sides of $C_{2}$ linearly scaled by the factor $n<1$ and $(1-n)$, respectively. Physically, we are assuming that the external inductance and resistance in each leg is scaled by the position of $C_{2}$ relative to $C_{1}$ and the coil, with $n$ approaching unity as $C_{2}$ is moved nearer to the coil.

A set of circuit equations for Fig. 1C are written through the application of Kirchoff's law to each loop. Rearranging the equations and adding a statement for the time-rate of change of charge on each capacitor yields the following coupled set of first-order ordinary differential equations:

$$
\begin{aligned}
\frac{d I_{1}}{d t} & =\frac{V_{1}-V_{2}-n R_{e} I_{1}}{n L_{0}}, \\
\frac{d I_{2}}{d t} & =\left\{L_{C} V_{2}+\left(L_{C} I_{3}+M I_{2}\right) \frac{d M}{d t}-(1-n) R_{e} L_{C} I_{2}-R_{p} M I_{3}\right\} /\left\{L_{C}\left[(1-n) L_{0}+L_{C}\right]-M^{2}\right\}, \\
\frac{d I_{3}}{d t} & =\frac{I_{2} \frac{d M}{d t}+M \frac{d I_{2}}{d t} I_{3} R_{p}}{L_{C}}, \\
\frac{d V_{1}}{d t} & =-\frac{I_{1}}{C_{1}}, \\
\frac{d V_{2}}{d t} & =\frac{I_{1}-I_{2}}{C_{2}},
\end{aligned}
$$

where $V_{1}$ and $V_{2}$ are the voltages on capacitors $C_{1}$ and $C_{2}$, respectively.

The inductance of a planar, spiral inductive coil coupled to a plasma current sheet is difficult to compute analytically. However, based on experimental measurements it has been found ${ }^{2}$ that the mutual inductance can be modeled using the simple function

$$
M=L_{C} \exp \left(-\frac{z}{2 z_{0}}\right),
$$

which, when differentiated results in the following equation that governs the changing mutual inductance of the circuit:

$$
\frac{d M}{d t}=-\frac{L_{C}}{2 z_{0}} \exp \left(-\frac{z}{2 z_{0}}\right) \frac{d z}{d t} .
$$

As the current sheet moves forward, it entrains and accelerates any enocuntered gas. The propellant mass in the current sheet as a function of time can be written as

$$
m(t)=m_{0}+\int_{t=0}^{t} \rho_{A} v_{z} d t,
$$

where $\rho_{A}=\rho_{A}(z(t))$ is the linear mass density distribution and $v_{z}$ is the sheet velocity. The term $m_{0}$ represents the initial mass of propellant in the sheet while the integral term represents the mass accumulated by the sheet as it moves away from the acceleration coil.

The momentum equation for this system can be written as

$$
\frac{L_{C} I_{2}^{2}}{2 z_{0}} \exp \left(-z / z_{0}\right)=\rho_{A} v_{z}^{2}+m(t) \frac{d v_{z}}{d t} .
$$

The left hand side represents the self-field electromagnetic force generated through the interaction of the current and the magnetic field. The first term on the right hand side in Eq. (5) is the momentum investment associated with 'snowplowing' propellant (i.e. accelerating the newly entrained propellant encountered by the sheet from rest to the sheet speed) while the second term involves further acceleration of the already entrained propellant.

The model lacks a plasma model to calculate temperature and resistivity and it does not contain any plasma ionization, or 'breakdown', model, instead simply assuming that there is plasma present at $t=0$. These shortcomings were previously discussed in more detail in Ref. [7]. As in that previous work, the purpose here is to use the model to search for relevant nondimensional scaling parameters and gauge their general effect on performance, instead of looking for an exact matching of experimental data to the performance model. However, we do note that even with the shortcomings, the 1-D, circuit-based acceleration model has shown good qualitative and quantitative agreement with experimental data. ${ }^{1,3}$ 


\section{B. Nondimensionalization of the Model}

The nondimensionalization strategy previously employed with success in Ref. [7] can also be used in this case. The following dimensionless terms are selected to aid in the conversion of the dimensional equations to a nondimensionalized set:

$$
\begin{aligned}
I_{1}^{*} & =\frac{1}{V_{0}} \sqrt{\frac{L_{0}}{C}} I_{1}, & I_{2}^{*} & =\frac{1}{V_{0}} \sqrt{\frac{L_{0}}{C}} I_{2} \\
I_{3}^{*} & =\frac{1}{V_{0}} \sqrt{\frac{L_{0}}{C}} I_{3} & t^{*} & =\frac{t}{\sqrt{L_{0} C}} .
\end{aligned}
$$

Other dimensionless terms that arise in the course of nondimensionalizing the equation set are:

$$
\begin{array}{ll}
z^{*}=\frac{z}{z_{0}}, & V_{1}^{*}=\frac{V_{1}}{V_{0}}, \quad V_{2}^{*}=\frac{V_{2}}{V_{0}}, \\
M^{*}=\frac{M}{L_{C}}, & v_{z}^{*}=\frac{\sqrt{L_{0} C}}{z_{0}} v_{z} .
\end{array}
$$

The current sheet mass can be nondimensionalized as

$$
m^{*}=m_{0}^{*}+\int_{0}^{t^{*}} \rho^{*} f\left(z^{*}\right) v_{z}^{*} d t^{*},
$$

where $m_{0}^{*}=m_{0} / m_{\mathrm{bit}}, \rho^{*}=\rho_{0} z_{0} / m_{\mathrm{bit}}$, and $m_{\mathrm{bit}}$ is the total propellant mass per pulse.

Using these nondimensional terms, equations (1), (3), (4), and (5) are written in dimensionless form as,

$$
\begin{aligned}
\frac{d I_{1}^{*}}{d t^{*}} & =\frac{1}{n}\left(V_{1}^{*}-V_{2}^{*}\right)-I_{1}^{*} \psi_{1} \\
\frac{d I_{2}^{*}}{d t^{*}} & =\left[L^{*} V_{2}^{*}+\left(M^{*} I_{2}^{*}+I_{3}^{*}\right) \frac{d M^{*}}{d t^{*}}-I_{3}^{*} M^{*} L^{*} \psi_{2}-(1-n) I_{2}^{*} L^{*} \psi_{1}\right] /\left[\left((1-n) L^{*}+1\right)-\left(M^{*}\right)^{2}\right](9 \mathrm{~b}) \\
\frac{d I_{3}^{*}}{d t^{*}} & =M^{*} \frac{d I_{2}^{*}}{d t^{*}}+I_{2}^{*} \frac{d M^{*}}{d t^{*}}-I_{3}^{*} L^{*} \psi_{2} \\
\frac{d V_{1}^{*}}{d t^{*}} & =-I_{1}^{*} \\
\frac{d V_{2}^{*}}{d t^{*}} & =\mathcal{C}\left(I_{1}^{*}-I_{2}^{*}\right) \\
\frac{d M^{*}}{d t^{*}} & =-\frac{1}{2} \exp \left(-\frac{z^{*}}{2}\right) v_{z}^{*} \\
\frac{d z^{*}}{d t^{*}} & =v_{z}^{*} \\
\frac{d v_{z}^{*}}{d t^{*}} & =\left[\alpha\left(I_{2}^{*}\right)^{2} \exp \left(-z^{*}\right)-\rho^{*} f\left(z^{*}\right)\left(v_{z}^{*}\right)^{2}\right] / m^{*} \\
\frac{d m^{*}}{d t^{*}} & =\rho^{*} f\left(z^{*}\right) v_{z}^{*}
\end{aligned}
$$

The initial conditions for solving the nondimensional set of equations are as follows.

$$
\begin{array}{ll}
I_{1}^{*}(0)=0, & M^{*}(0)=1, \\
I_{2}^{*}(0)=0, & z^{*}(0)=0, \\
I_{3}^{*}(0)=0, & v_{z}^{*}(0)=0, \\
V_{1}^{*}(0)=1, & m^{*}(0)=\frac{m_{0}}{m_{\text {bit }}} . \\
V_{2}^{*}(0)=0, &
\end{array}
$$




\section{Scaling Parameters}

During the course of nondimensionalization, several new terms appear in Eqs. (9). These new terms are the relevant scaling parameters of the system of equations and are defined as

$$
\begin{array}{ll}
\mathcal{C}=\frac{C_{1}}{C_{2}}, & L^{*}=\frac{L_{0}}{L_{C}}, \quad \alpha=\frac{C_{1}^{2} V_{0}^{2} L_{C}}{2 m_{\text {bit }} z_{0}^{2}}, \\
\psi_{1}=R_{e} \sqrt{\frac{C_{1}}{L_{0}}}, & \psi_{2}=R_{p} \sqrt{\frac{C_{1}}{L_{0}}},
\end{array}
$$

which are similar to the parameters previously found for the single capacitor case. ${ }^{7}$ We proceed with a brief discussion of the meanings of the scaling parameters.

\section{Inductance Ratio $L^{*}$}

A pulsed inductive accelerator circuit possesses an external inductance $L_{0}$ and an acceleration coil inductance $L_{C}$. During a current pulse the moving plasma increases the circuit's inductance from $L_{0}$ to $L_{0}+L_{C}$ (i.e., $\left.L_{C}=\Delta L\right)$. The fractional change of inductance, $\left(L^{*}\right)^{-1}=\Delta L / L_{0}$, in a pulsed electromagnetic accelerator provides a measure of efficiency, ${ }^{9}$ as this ratio is indicative of the fraction of energy that can be deposited into electromagnetic acceleration of the gas. In an efficient pulsed inductive accelerator the value of $L^{*}$ must be much less than unity or $L_{C} \gg L_{0}$.

\section{Dynamic Impedance Parameter $\alpha$}

The dynamic impedance parameter $\alpha$ can be recast as the product of several important ratios:

$$
\alpha=\frac{C_{1}^{2} V_{0}^{2} L_{C}}{2 m_{\mathrm{bit}} z_{0}^{2}}=\frac{1}{8 \pi^{2}} \frac{C_{1} V_{0}^{2} / 2}{m_{\mathrm{bit}} v_{z}^{2} / 2} L^{*}\left(\frac{2 \pi \sqrt{L_{0} C_{1}}}{L_{0} / \dot{L}}\right)^{2}
$$

where $\dot{L}$ is the dynamic impedance, defined as $v_{z} L^{\prime}$, and $L^{\prime}$ is the effective inductance per unit length, equal to $L_{C} / z_{0}$. The ratio of the initial stored energy $\left(C_{1} V_{0}^{2} / 2\right)$ to the plasma kinetic energy $\left(m_{\text {bit }} v_{z}^{2} / 2\right)$ is recognized as the inverse of thrust efficiency $\eta_{t}^{-1}$ and will always be greater than one. The final term is the ratio of the natural period of the driving external circuit, $2 \pi \sqrt{L_{0} C_{1}}$, to the time interval, $L_{0} / \dot{L}$, over which the plasma's motion increases the inductance of the circuit by one unit of $L_{0}$. The former is the timescale on which the external circuit naturally operates, while the latter is the timescale on which the current sheet remains electromagnetically coupled to the acceleration coil. For a given configuration, there exists an optimum value of $\alpha$ where the electromagnetic coupling timescale is matched to the frequency of the external circuit, allowing for optimum transfer of stored electrical energy into directed kinetic energy. ${ }^{7}$

\section{Circuit Parameters $\psi_{1}, \psi_{2}$ and $\mathcal{C}$}

The critical resistance ratios $\psi_{1}$ and $\psi_{2}$ were shown in previous work to control the nature of the current waveforms. ${ }^{7}$ The new parameter $\mathcal{C}$ also has an effect on the nature of the current waveforms. Proceeding with an analysis similar to that performed in Ref. [7], we search for limiting-case solutions to Eqns. (9a)-(9e). Decoupling the current sheet dynamics (i.e., the acceleration and sheet motion) from the problem allows us to apply the condition

$$
M^{*}=1, \quad \frac{d M^{*}}{d t^{*}}=0,
$$

which simplify the circuit equations. Under these assumptions, the circuit equations can be combined and rewritten as

$$
\begin{aligned}
\frac{d^{2} I_{1}^{*}}{d t^{* 2}}+\psi_{1} \frac{d I_{1}^{*}}{d t^{*}}+\frac{(\mathcal{C}+1)}{n} I_{1}^{*} & =\frac{\mathcal{C}}{n} I_{2}^{*}, \\
(1-n) \frac{d^{2} I_{2}^{*}}{d t^{* 2}}+(1-n) \psi_{1} \frac{d I_{2}^{*}}{d t^{*}}+\mathcal{C} I_{2}^{*} & =\mathcal{C} I_{1}^{*}-\psi_{2} \frac{d I_{3}^{*}}{d t^{*}} \\
\frac{d I_{2}^{*}}{d t^{*}}-\frac{d I_{3}^{*}}{d t^{*}} & =L^{*} \psi_{2} I_{3}^{*}
\end{aligned}
$$


If the right side of the third equation in (13) is small, then the induced current in the plasma mirrors the current in the coil

$$
I_{3}^{*} \approx I_{2}^{*} .
$$

This is the case where the coil and plasma currents are well-coupled, which is expected in efficient accelerators.

The third equation of (13) can be substituted into the second to yield

$$
(1-n) \frac{d^{2} I_{2}^{*}}{d t^{* 2}}+\left[(1-n) \psi_{1}+\psi_{2}\right] \frac{d I_{2}^{*}}{d t^{*}}+\mathcal{C} I_{2}^{*}=\mathcal{C} I_{1}^{*}-\psi_{2}^{2} L^{*} I_{3}^{*}
$$

This second-order non-linear ODE is similar to the one found in Ref. [7]. If the last term on the right-hand side is comparitively small, then the forcing term contains only $I_{1}^{*}$, which will never be small and cannot be neglected. We can gain insight by writing the homogeneous solution

$$
I_{2}^{*}=A_{0} \exp \left(-\Psi t^{*}\right) \sin \left(\left(\frac{\mathcal{C}}{(1-n)}-\Psi^{2}\right)^{1 / 2} t^{*}\right),
$$

where

$$
\Psi \equiv\left(\psi_{1}+\frac{\psi_{2}}{(1-n)}\right) / 2
$$

In this case, the damping is a function of the critical resistance ratios while the natural frequency is additionally a function of the parameter $\mathcal{C}$.

The term on the right hand side of the first equation in (13) will also never be small. However, we can write the homogeneous solution for $I_{1}^{*}$ as

$$
I_{1}^{*}=B_{0} \exp \left(-\frac{\psi_{1}}{2} t^{*}\right) \sin \left(\left(\frac{(\mathcal{C}+1)}{n}-\frac{\psi_{1}^{2}}{4}\right)^{1 / 2} t^{*}\right) .
$$

Though these are only the homogeneous solutions to the simplified electrical response equations, there are several observations that can be made as a result of this exercise. The parameters $\psi_{1}, \psi_{2}$, and $\mathcal{C}$ control the electrical responses in the circuit. Increasing the parameter $\mathcal{C}$ increases the ringing frequency of both $I_{1}^{*}$ and $I_{2}^{*}$, with the frequencies approaching each other as $\mathcal{C}$ becomes very large. To neglect the non-linear terms presented by the current $I_{3}^{*}$, the values of $L^{*}$ and $\psi_{2}$ must be such that

$$
\begin{aligned}
\psi_{2}^{2} L^{*} & \ll 1, \\
\psi_{2} L^{*} & \ll 1 .
\end{aligned}
$$

Finally, Eq. (9e) can be used to show that as $\mathcal{C}$ approaches infinity, the derivative $d V_{2}^{*} / d t^{*}$ will only remain bounded if $I_{1}^{*}=I_{2}^{*}$. Physically, this is the case where $C_{2}$ is removed from the system, permitting the recovery of the response for the circuit shown in Fig. 1B.

\section{Nondimensional Solutions}

\section{A. Solution Strategy}

The set of coupled first-order ODEs given in Eqs. (9) can be solved numerically once the mass distribution and the set of scaling parameters given in Eqs. (11) are specified. The performance metrics chosen for this study are the exhaust velocity, $v_{z}^{*}$, and the thrust efficiency, which is written in terms of nondimensional parameters as

$$
\eta_{\mathrm{t}}=\frac{m^{*}\left(v_{z}^{*}\right)^{2}}{2 L^{*} \alpha}
$$

Note that this corrects the equation given for efficiency in Ref. [7] where the square term was left off $v_{z}^{*}$ (though it was included in the analysis contained in the reference).

In solving any set of first-order (in time) differential equations, it is important to know when the time histories of the computed variables (specifically $v_{z}^{*}$ in our case) should be queried to calculate performance. This question is, in fact, critical to the evaluation of these accelerators. For our non-dimensional model, the integration period will end when one of the following two conditions is reached: 
1. The end of the first half-cycle of the accelerator coil discharge is reached and the current $I_{1}^{*}$ reverses in sign.

2. The sheet travels three characteristic lengths, $z^{*}=3$.

The first condition is based on the fact that when the accelerator current goes through zero, it is going though a point of high $d I / d t$. While the acceleration model doesn't incorporate any ionization physics, it is well known that a new current sheet can form at the face of the coil during current reversals, causing what is known as a "crowbar discharge". If this occurs, the initial current sheet ceases to undergo acceleration. If at the time of current reversal the final current sheet mass $m_{f}^{*}$ is less than the injected propellant mass bit $m_{\text {bit }}^{*}$, conservation of momentum can be used to correct the end-of-calculation final value of velocity $\left(v_{z}^{*}\right)_{f}$ to give the velocity as

$$
v_{z}^{*}=\frac{m_{f}^{*}}{m_{\mathrm{bit}}^{*}}\left(v_{z}^{*}\right)_{f}
$$

The second condition stems from the existence of a finite axial distance between the current sheet and the coil, beyond which the two are essentially decoupled electromagnetically. The separation distance $z^{*}=3$ is chosen as our cutoff for electromagnetic coupling as it represents an inductance change in the circuit of $95 \%$ of the coil inductance. Above this cutoff value, the integration yields no significant change to the calculated performance.

For the present study, a triangular (linearly decreasing) mass distribution is used. This is given by

$$
\rho_{A}^{*}= \begin{cases}\rho_{0}^{*}\left(1-z^{*} / \delta_{m}^{*}\right) & z^{*} \leq \delta_{m}^{*} \\ 0 & z^{*}>\delta_{m}^{*},\end{cases}
$$

where $\rho_{0}^{*}=2 / \delta_{m}^{*}, \delta_{m}^{*}=\delta_{m} / z_{0}$, and $\delta_{m}$ is the (dimensional) depth of the propellant layer.

\section{B. Solutions}

\section{Electrical Response as a Function of $\mathcal{C}$ and $n$}

We first present representative waveforms showing the electrical behavior of the accelerator as a function of $\mathcal{C}$ in Fig. 2 and $n$ in Fig. 3. The former gauges the effect of the capacitance ratio $\mathcal{C}$ while the latter gives insight into the effect of the position of capacitor $C_{2}$ in the line relative to $C_{1}$ and the coil. Waveforms for $I_{1}^{*}, I_{2}^{*}$ and $d I_{2}^{*} / d t^{*}$ are presented, where the latter provides a measure of the inductive voltage across the coil and are being used in this study as proxies for the ability of the coil to inductively ionize propellant.

In Fig. 2 we observe that currents $I_{1}^{*}$ and $I_{2}^{*}$ have the standard damped-sinusoidal response of an $L R C$ circuit with a higher-frequency waveform superimposed on the response. The modulation in all the waveforms increases in frequency and decreases in amplitude as $\mathcal{C}$ is increased. For $\mathcal{C}=1$ we observe a nearly resonant energy transfer between $C_{1}$ and $C_{2}$ with the rise rate of the current in the coil relatively low in comparison with the other cases. As $\mathcal{C}$ is increased to 100 , the maximum value of $d I_{2}^{*} / d t^{*}$ approaches 2.

The changing value of $n$ appears to most affect the response of the current $I_{1}^{*}$ in Fig. 3, with the amplitude of the oscillation superimposed on the wave decreasing with increasing $n$. We observe that for smaller $n$ values $d I_{2}^{*} / d t^{*}$ oscillates much longer and, like $I_{1}^{*}$, with greater amplitude, having the greatest peak value for the smallest $n$ displayed. It is easiest to observe in Fig. 3G,H that the overall average behavior of $I_{1}^{*}$ and $I_{2}^{*}$ are roughly equivalent $\left(I_{1}^{*} \approx I_{2}^{*}\right)$ for the cases where $\mathcal{C}$ equals 100 , which was predicted in the limiting case solutions for $\mathcal{C}$ sufficiently large. The form and magnitude of the waveform for $I_{2}^{*}$ does not appear to change much with $n$, implying that the acceleration process is generally unaffected by the position of $C_{2}$ in the line. For the remainder of this study we use $n=0.2$ to obtain greater current rise rates.

\section{Nondimensional Performance}

Contours of constant computed performance $\left(\eta_{\mathrm{t}}, v_{z}^{*}\right)$ and $d I_{2}^{*} / d t^{*}$ found through solving the governing equations for a range of $\mathcal{C}$ and $\alpha$ values are presented in Fig. 4A-C. For a fixed $\mathcal{C}$ the efficiency possesses a local maximum with respect to $\alpha$, which is consistent with the interpretation of $\alpha$ as a dynamic impedance matching parameter. The efficiency at a fixed $\alpha$ initially increases with increasing $\mathcal{C}$, eventually asymptoting at $\mathcal{C}$ between 10 and 100. The velocity increases with $\alpha$ and like the efficiency it also asymptotes as $\mathcal{C}$ is 


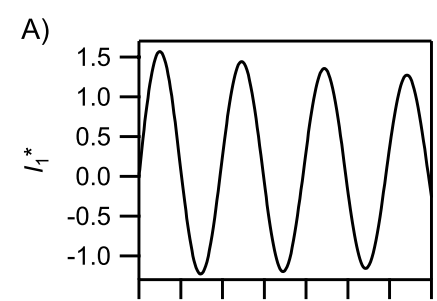

D)

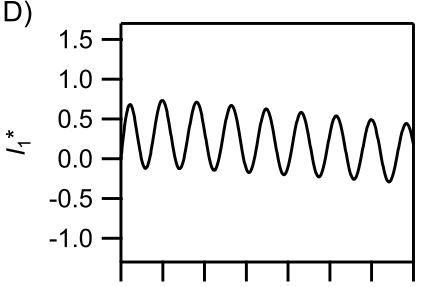

G)

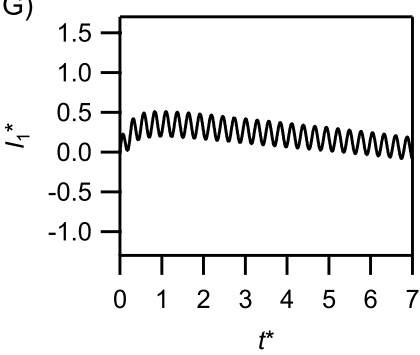

B)

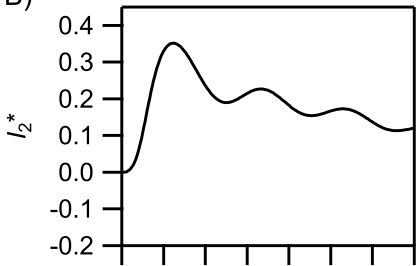

E)

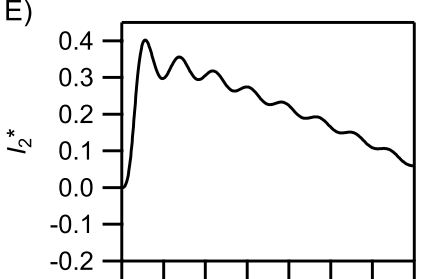

H)

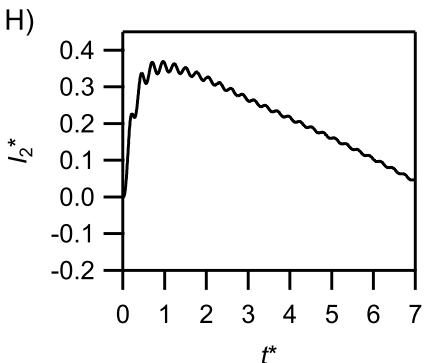

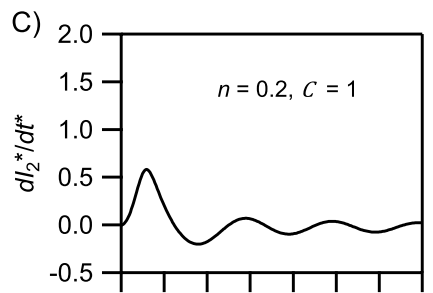

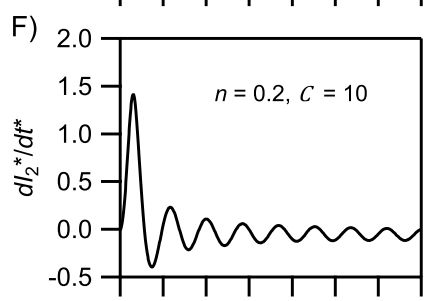

l)

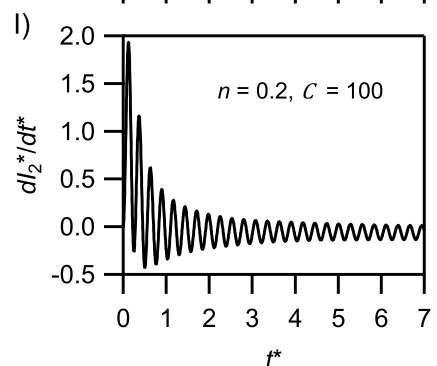

Figure 2. Waveforms for $I_{1}^{*}, I_{2}^{*}$, and $d I_{2}^{*} / d t^{*}$ showing the variation in each for A)-C) $\left.n=0.2, \mathcal{C}=1, \mathbf{D}\right)-\mathbf{F}$ ) $n=0.2, \mathcal{C}=10$, and $\mathbf{G})-\mathbf{I}) n=0.2, \mathcal{C}=100$.
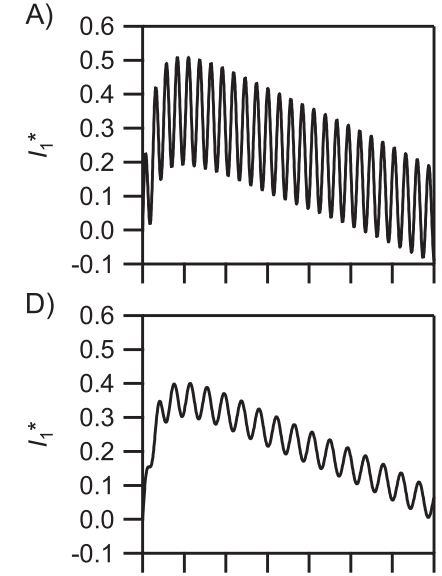

G)

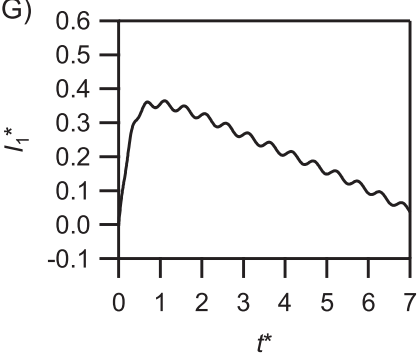

B)

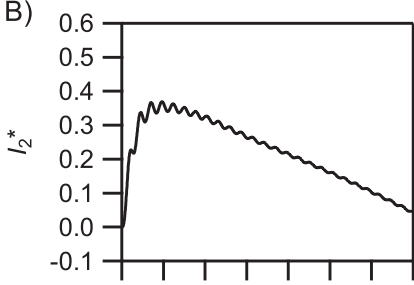

E)

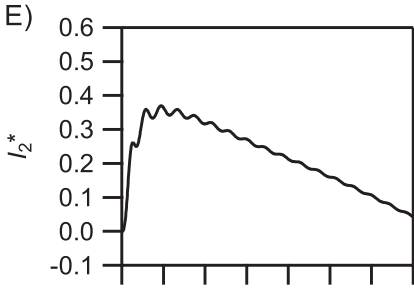

H)

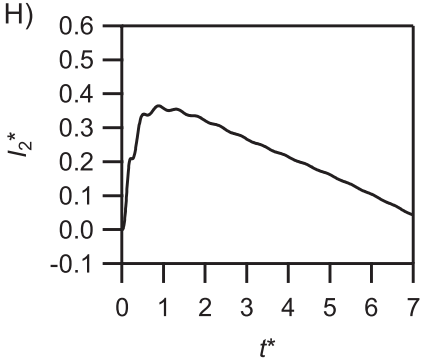

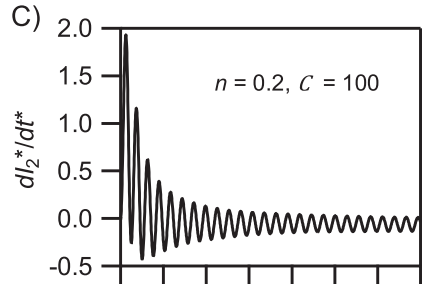

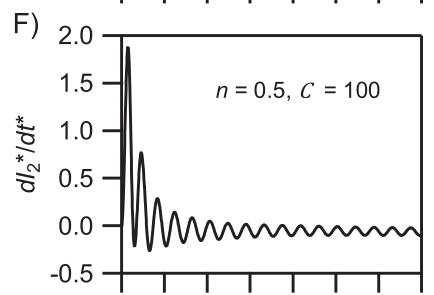

।)

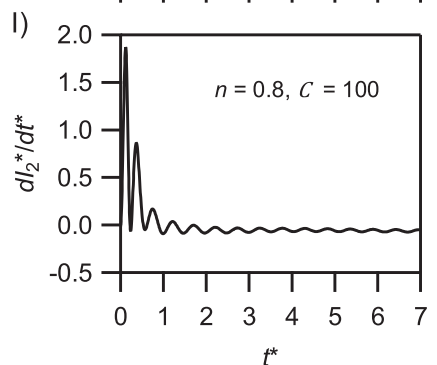

Figure 3. Waveforms for $I_{1}^{*}, I_{2}^{*}$, and $d I_{2}^{*} / d t^{*}$ showing the variation in each for A)-C) $\left.n=0.2, \mathcal{C}=100, \mathbf{D}\right)-\mathbf{F}$ ) $n=0.5, \mathcal{C}=100$, and $\mathbf{G})-\mathbf{I}) n=0.8, \mathcal{C}=100$. 
increased. The current rise rate is a weak function of $\alpha$, but increases very quickly as $\mathcal{C}$ is increased until asymptoting just below a value of $d I_{2}^{*} / d t^{*}$ equal to 2 .

It is worth comparing solutions to the two-capacitor case (Fig. 1C) with solutions to the single-capacitor case (Fig. 1A,B) to gauge the effect of the added capacitor $C_{2}$ on accelerator performance. Curves of computed performance $\left(\eta_{\mathrm{t}}, v_{z}^{*}\right)$ and the maximum value of $d I_{2}^{*} / d t^{*}$ for $\mathcal{C}$ equal to $1.5,5,10$, and 100 are presented in Fig. 4D-F. An additional curve on each graph appears for the single-capacitor case (with the maximum value of $d I_{1}^{*} / d t^{*}$ given in Fig. $4 \mathrm{~F}$ ). We observe that there is not a large difference in the terminal exhaust velocity $v_{z}^{*}$ of the single and two-capacitor cases for any value of $\mathcal{C}$. The efficiency graph shows that the two-capacitor case asymptotes to the single-capacitor solution as $\mathcal{C}$ approaches 100 , implying that a capacitor $C_{2}$ can be added without detrimentally affected thruster performance. Additionally, as $\mathcal{C}$ is increased the maximum current rise rate through the coil in the two-capacitor case asymptotes to a value that is twice the single-capacitor case.
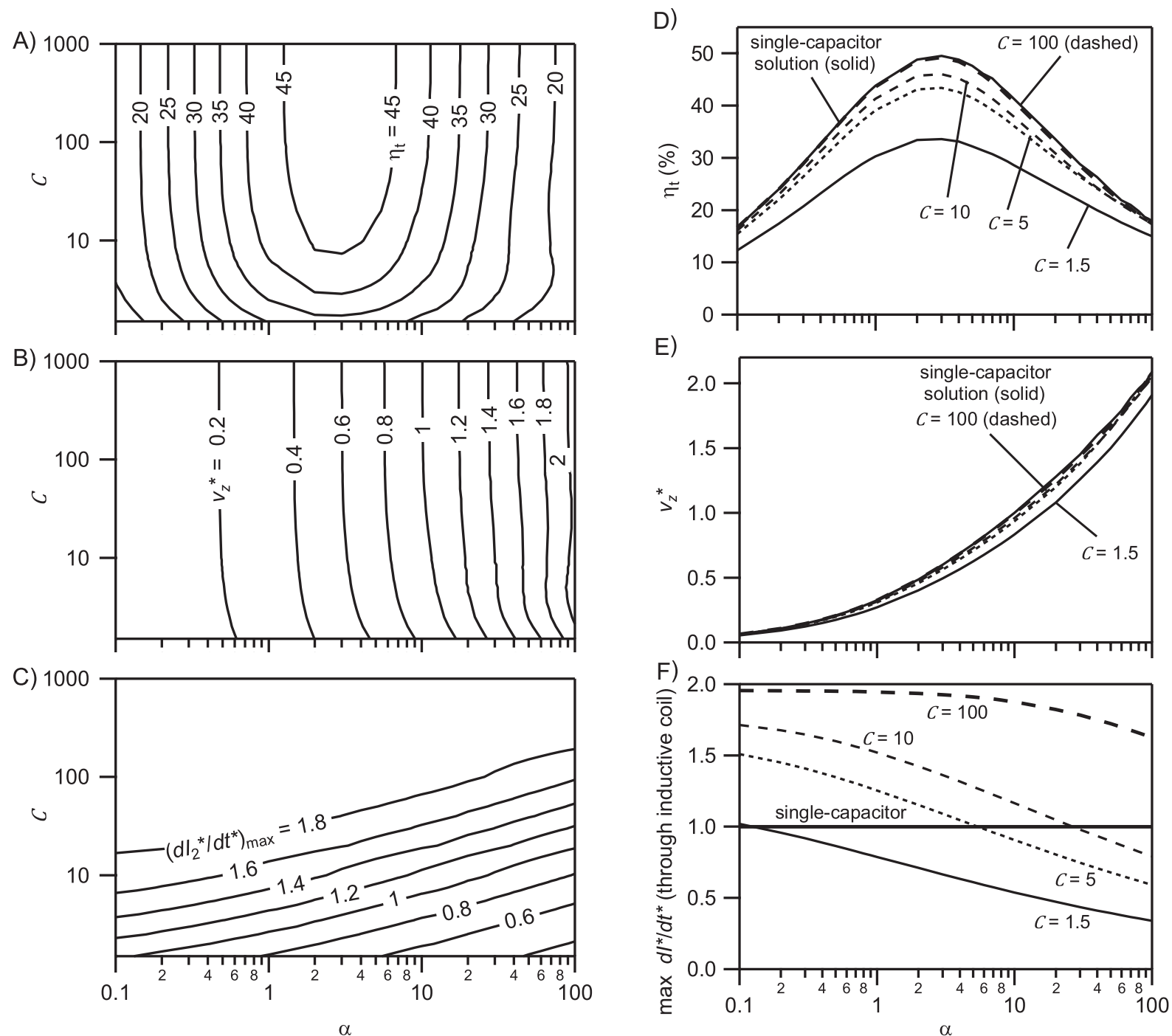

Figure 4. Contour plots of A) efficiency, B) nondimensional velocity, and C) maximum value of $d I_{2}^{*} / d t^{*}$ as a function of varying $\mathcal{C}$ and $\alpha$. Values of $\psi_{1}, \psi_{2}$, and $L^{*}$ are $0.05,0.13$, and 0.121 , respectively. Comparison of D) efficiency, E) nondimensional velocity, and F) maximum $d I^{*} / d t^{*}$ through the inductive coil as a function of $\alpha$ for various values of $\mathcal{C}$ and for the single-capacitor case. The values of maximum $d I_{2}^{*} / d t^{*}$ are given in F) except for the single capacitor case, where the maximum $d I_{1}^{*} / d t^{*}$ is shown. 


\section{Discussion}

The data presented in the previous section helped gauge the effect on performance that the addition of capacitor $C_{2}$ would have. Comparing the efficiency for the single and two-capacitor cases demonstrates that the performance above a certain value of $\mathcal{C}$ is not adversely affected by the the addition of the capacitor $C_{2}$. This result is somewhat expected because the overall behavior of current $I_{2}^{*}$ (seen in Fig. 3) is relatively well-behaved, with only a small modulation superimposed on top of the main waveform, as opposed to the much larger excursions observed in Fig. 2 for smaller values of $\mathcal{C}$. In addition, at $\mathcal{C}$ of 100 the waveforms for $I_{1}^{*}$ and $I_{2}^{*}$ are approximately equivalent, implying that the presence of the capacitor $C_{2}$ is having little effect on the overall current through the inductive coil that is inducing fields in the plasma and acting on the gas to accelerate it.

As $\mathcal{C}$ is increased, the value of $d I_{2}^{*} / d t^{*}$ asymptotes to twice the value of $d I^{*} / d t^{*}$ through the coil in the single capacitor case. The reason for this can best be understood by focusing on the first current loop $\left(I_{1}\right)$ in Fig. $1 \mathrm{C}$. If there is no resistance in the loop, when $C_{1}=C_{2}(\mathcal{C}=1)$, the charge from $C_{1}$ transfers completely to $C_{2}$, with the current $I_{1}^{*}$ peaking when the voltage on each capacitor is equal and going to zero as the voltage on $C_{2}$ equals the original voltage on $C_{1}$. Consequently, the voltage across the coil is still roughly the same value as it would have been if $C_{2}$ was not present. This neglects resistive losses and doesn't account for the fact that much of the charge, instead of going through the coil from $C_{2}$, instead transfers back to $C_{1}$ robbing potential current and $d I_{2}^{*} / d t^{*}$ through the coil (a situation illustrated in Figs. 2A-C.).

We can best understand the case where $C_{1} \gg C_{2}$ by further simplifying current loop $I_{1}^{*}$ so it consists only of $C_{1}, C_{2}$, and $n L_{0}$. (Neglecting the current loops $I_{2}^{*}$ and $I_{3}^{*}$ and their associated lumped electrical elements does not detract from the reasoning that follows and serves to simplify the explanation.) Initially, the voltage on $C_{1}$ changes very little as the discharge progresses. The maximum current $I_{1}^{*}$, corresponding to $d I_{1}^{*} / d t^{*}=0$, occurs when the voltage on $C_{2}$ is approximately equal to the initial charge voltage on $C_{1}$. As time progresses further the voltage on $C_{2}$ continues to rise, eventually reaching a theoretical maximum value that is approximately twice the initial voltage on $C_{1}$ when the current $I_{1}^{*}=0$ and the value of $d I_{1}^{*} / d t^{*}$ is a local minimum. Translating this to the full circuit, we observe that this would imply a voltage across the coil that is approximately twice the voltage possible in the single capacitor case, yielding a commensurate doubling of $d I^{*} / d t^{*}$ through the coil.

\section{Conclusions}

We have used a nondimensional pulsed inductive acceleration model to study the effects of adding a second, parallel capacitor into the circuit topology. Inserting the second capacitor into the system was motivated by limited data showing that the voltage appearing across the accelerator coil could be increased significantly over the initial charge voltage. Increasing the voltage across the coil has been shown to be important in the process of inductively ionizing the propellant. The nondimensionalization of the modified model led to identification of new scaling parameters that are unique to the particular topology. The physical meanings of the new parameters and their effects on accelerator performance were explored through a series of theoretical arguments and numerical simulations. The value of current rise rate through the coil is used as a proxy measure for the voltage across the coil, with higher values of $d I / d t$ potentially yielding better inductive propellant ionization. The following insights were gained in this study.

- As in the single capacitor case, there exists a value of the dynamic impedance parameter $\alpha$ for which thrust efficiency is maximized. This optimum corresponds to a matching of the driving circuits natural oscillation time scale to the residence time scale of the current sheet in the acceleration zone.

- The efficiency and maximum $d I_{2}^{*} / d t^{*}$ through the coil suffer for lower values of capacitance ratio $\mathcal{C}$, with much of the charge transferring back and forth from $C_{1}$ to $C_{2}$ instead of going through the coil and performing work on the propellant.

- The efficiency of the two-capacitor system approaches the theoretical efficiency of the single capacitor topology for $\mathcal{C} \gg 1$, with the commensurate value of $d I_{2}^{*} / d t^{*}$ asymptoting to a value twice as large as that possible in the single capacitor case.

- As $\mathcal{C}$ is increased, the gross behavior of the current waveforms for $I_{1}^{*}$ and $I_{2}^{*}$ match. The differences in these waveforms consists of a high-frequency modulation overlayed on the gross waveform. For a given 
$\mathcal{C}$ the modulation is a function of $n$, which represents the position of $C_{2}$ relative to $C_{1}$ and the coil. The value of $n$ greatly affects the level of modulation on $I_{1}^{*}$ but only slightly affects $I_{2}^{*}$, implying very little change in the acceleration process as a function of $n$.

\section{Acknowledgments}

We appreciate the continued MSFC management support of Mr. Jim Martin and Ms. Mary Beth Koelbl. We gratefully acknowledge several helpful conversations with Mr. J. Boise Pearson during the preparation of this work. Author J.V. Balla received support from the Ohio Space Grant Consortium for his effort on this work. This work was completed under NASA's Advanced In-Space Propulsion program managed by Dr. Michael LaPointe.

\section{References}

\footnotetext{
${ }^{1}$ K.A. Polzin, "Comprehensive review of planar pulsed inductive plasma thruster research and technology," J. Propuls. Power, 27(3):513, 2011.

${ }^{2}$ R.H. Lovberg and C.L. Dailey, "Large inductive thruster performance measurement," AIAA J., 20(7):971, 1982.

${ }^{3}$ C.L. Dailey and R.H. Lovberg, "The PIT MkV Pulsed Inductive Thruster," TRW Systems Group, Tech. Rep. NASA CR-191155, Jul. 1993.

${ }^{4}$ E.Y. Choueiri and K.A. Polzin, "Faraday Accelerator with Radio-Frequency Assisted Discharge (FARAD)," J. Propuls. Power, 22(3):611, 2006.

${ }^{5}$ K.A. Polzin, "Scaling and Systems Considerations in Pulsed Inductive Plasma Thrusters," IEEE Trans. Plasma Sci., 36(5):2189, 2008.

${ }^{6}$ C.L. Dailey, "Pulsed Electromagnetic Thruster," TRW Systems Group, AFRPL-TR-71-107, 1971.

${ }^{7}$ K.A. Polzin and E.Y. Choueiri, "Performance optimization criteria for pulsed inductive plasma acceleration," IEEE Trans. Plasma Sci., 34(3):945, 2006.

${ }^{8}$ K.A. Polzin, Faraday Accelerator with Radio-Frequency Assisted Discharge (FARAD), Ph.D. Dissertation, 3147-T, Princeton Univ., Princeton, NJ, 2006.

${ }^{9}$ R.H. Lovberg, B.R. Hayworth, and T. Gooding, "The Use of a Coaxial Plasma Gun for Plasma Propulsion," Tech. Rep. AE62-0678, May 1962, G.D. Convair.
} 


\section{Effect of an Additional, Parallel Capacitor on Pulsed Inductive Plasma Accelerator Performance}

$32^{\text {nd }}$ International Electric Propulsion Conference

IEPC Paper 2011-160

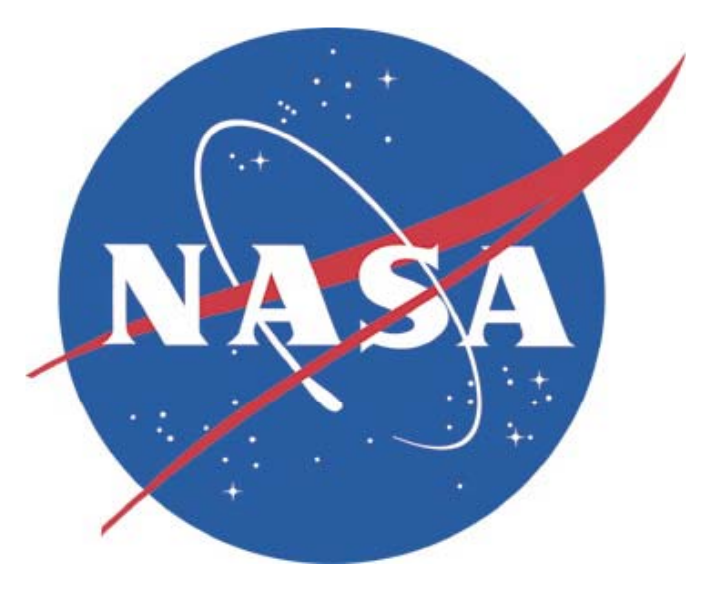

Kurt Polzin, Amy Sivak, Joseph Balla NASA-Marshall Space Flight Center 


\section{Introduction}

- Typically pulsed inductive thrusters limited to a simple RLC ringing circuit

- A single data point (from 1971) indictated adding a second capacitor in the system downstream of the main bank offered potential performance improvement and a greater inductive voltage drop across the coil

- Implies higher $d I / d t$ through the coil

- Greater $d I / d t$ through the coil could result in more efficient and/or complete inductive breakdown/ionization of the propellant

- In this study

- Model the system with a second capacitor, nondimensionalizing to explore the global properties

- Calculate the effect of the second capacitor on performance

- Calculate $d l / d t$ through the coil, using it's value as a proxy measurement for the ability to inductively ionize the propellant 


\section{Circuit Model}

- Two current loops, coupled through the inductive acceleration coil

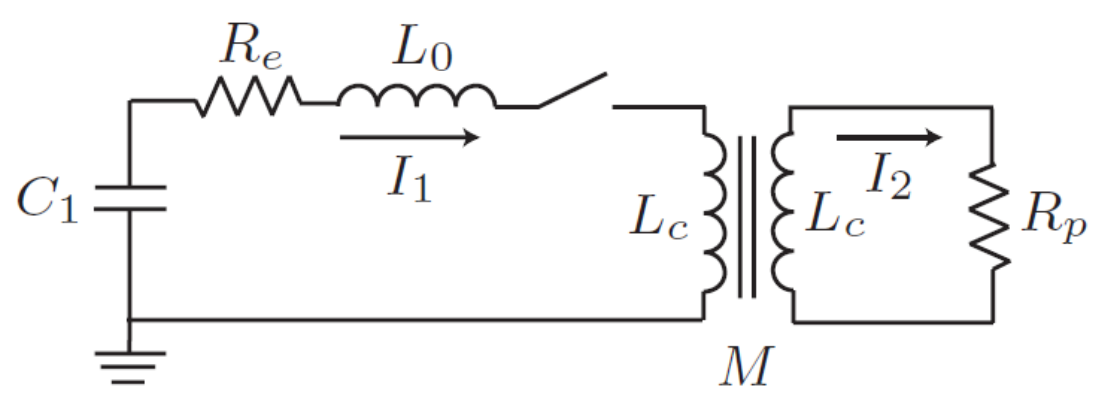

- Equivalent circuit

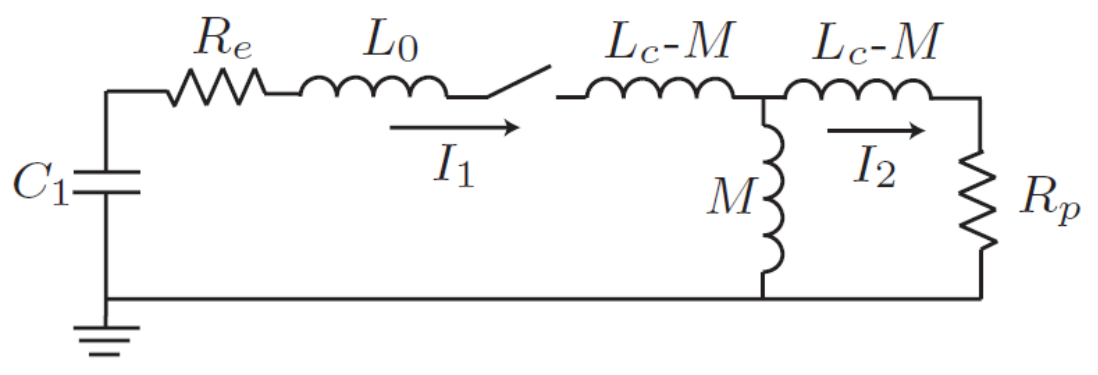




\section{Circuit Model w/Additional Capacitor}

- $\quad$ Added new capacitor $C_{2}$

- Stray inductance $L_{0}$, external resistance $R_{\mathrm{e}}$ split between loops for $I_{1}$ and $I_{2}$

- Constant $n$ associated with how close $C_{2}$ is to $C_{1}$

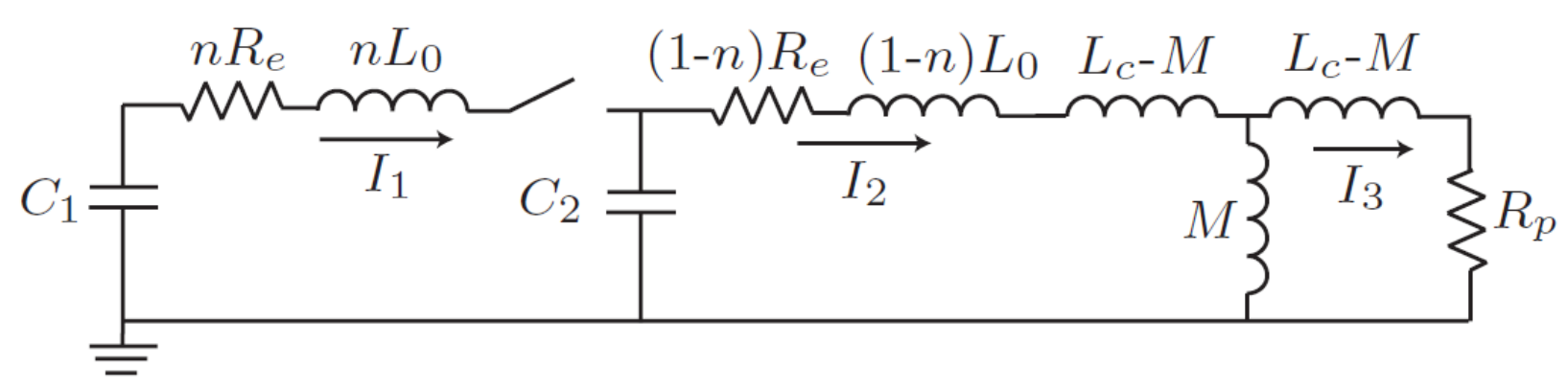




\section{Circuit Equations}

- Governing equations for current (from Kirchoff's law)

$$
\begin{aligned}
\frac{d I_{1}}{d t} & =\frac{V_{1}-V_{2}-n R_{e} I_{1}}{n L_{0}}, \\
\frac{d I_{2}}{d t} & =\left\{L_{C} V_{2}+\left(L_{C} I_{3}+M I_{2}\right) \frac{d M}{d t}-(1-n) R_{e} L_{C} I_{2}-R_{p} M I_{3}\right\} /\left\{L_{C}\left[(1-n) L_{0}+L_{C}\right]-M^{2}\right\}, \\
\frac{d I_{3}}{d t} & =\frac{I_{2} \frac{d M}{d t}+M \frac{d I_{2}}{d t} I_{3} R_{p}}{L_{C}},
\end{aligned}
$$

- Governing equations for voltage on the capacitors (from charge conservation)

$$
\frac{d V_{1}}{d t}=-\frac{I_{1}}{C_{1}}, \quad \frac{d V_{2}}{d t}=\frac{I_{1}-I_{2}}{C_{2}},
$$

- Governing equation for the mutual inductance (empirical model)

$$
\frac{d M}{d t}=-\frac{L_{C}}{2 z_{0}} \exp \left(-\frac{z}{2 z_{0}}\right) \frac{d z}{d t}
$$




\section{Acceleration Model Equations}

- Mass Accumulation Equation (snowplow model)

$$
m(t)=m_{0}+\int_{t=0}^{t} \rho_{A} v_{z} d t
$$

- Momentum Equation

$$
\begin{gathered}
\frac{L_{C} I_{2}^{2}}{2 z_{0}} \exp \left(-z / z_{0}\right)=\rho_{A} v_{z}^{2}+m(t) \frac{d v_{z}}{d t} \\
\text { EM Force } \\
\text { Acceleration }
\end{gathered}
$$




\section{Non-dimensionalization}

- Initial non-dimensional terms

$$
\begin{array}{rlrl}
I_{1}^{*} & =\frac{1}{V_{0}} \sqrt{\frac{L_{0}}{C}} I_{1}, & I_{2}^{*}=\frac{1}{V_{0}} \sqrt{\frac{L_{0}}{C}} I_{2} \\
I_{3}^{*}=\frac{1}{V_{0}} \sqrt{\frac{L_{0}}{C}} I_{3} & t^{*}=\frac{t}{\sqrt{L_{0} C}} .
\end{array}
$$

- Additional terms

$$
\begin{gathered}
z^{*}=\frac{z}{z_{0}}, \quad V_{1}^{*}=\frac{V_{1}}{V_{0}}, \quad V_{2}^{*}=\frac{V_{2}}{V_{0}}, \\
M^{*}=\frac{M}{L_{C}}, \quad v_{z}^{*}=\frac{\sqrt{L_{0} C}}{z_{0}} v_{z} \\
m_{0}^{*}=m_{0} / m_{\mathrm{bit}} \quad \rho^{*}=\rho_{0} z_{0} / m_{\mathrm{bit}}
\end{gathered}
$$




\section{Non-dimensional equations}

$$
\begin{aligned}
\frac{d I_{1}^{*}}{d t^{*}} & =\frac{1}{n}\left(V_{1}^{*}-V_{2}^{*}\right)-I_{1}^{*} \psi_{1} \\
\frac{d I_{2}^{*}}{d t^{*}} & =\left[L^{*} V_{2}^{*}+\left(M^{*} I_{2}^{*}+I_{3}^{*}\right) \frac{d M^{*}}{d t^{*}}-I_{3}^{*} M^{*} L^{*} \psi_{2}-(1-n) I_{2}^{*} L^{*} \psi_{1}\right] /\left[\left((1-n) L^{*}+1\right)-\left(M^{*}\right)^{2}\right] \\
\frac{d I_{3}^{*}}{d t^{*}} & =M^{*} \frac{d I_{2}^{*}}{d t^{*}}+I_{2}^{*} \frac{d M^{*}}{d t^{*}}-I_{3}^{*} L^{*} \psi_{2} \\
\frac{d V_{1}^{*}}{d t^{*}} & =-I_{1}^{*} \\
\frac{d V_{2}^{*}}{d t^{*}} & =\mathcal{C}\left(I_{1}^{*}-I_{2}^{*}\right) \\
\frac{d M^{*}}{d t^{*}} & =-\frac{1}{2} \exp \left(-\frac{z^{*}}{2}\right) v_{z}^{*} \\
\frac{d z^{*}}{d t^{*}} & =v_{z}^{*} \\
\frac{d v_{z}^{*}}{d t^{*}} & =\left[\alpha\left(I_{2}^{*}\right)^{2} \exp \left(-z^{*}\right)-\rho^{*} f\left(z^{*}\right)\left(v_{z}^{*}\right)^{2}\right] / m^{*} \\
\frac{d m^{*}}{d t^{*}} & =\rho^{*} f\left(z^{*}\right) v_{z}^{*}
\end{aligned}
$$




\section{Initial Conditions / Scaling Parameters}

\begin{tabular}{|ll|}
\hline \multicolumn{2}{|c|}{ Initial conditions } \\
$I_{1}^{*}(0)=0$, & $M^{*}(0)=1$, \\
$I_{2}^{*}(0)=0$, & $z^{*}(0)=0$, \\
$I_{3}^{*}(0)=0$, & $v_{z}^{*}(0)=0$, \\
$V_{1}^{*}(0)=1$, & $m^{*}(0)=\frac{m_{0}}{m_{\text {bit }}}$. \\
$V_{2}^{*}(0)=0$, & \\
\hline
\end{tabular}

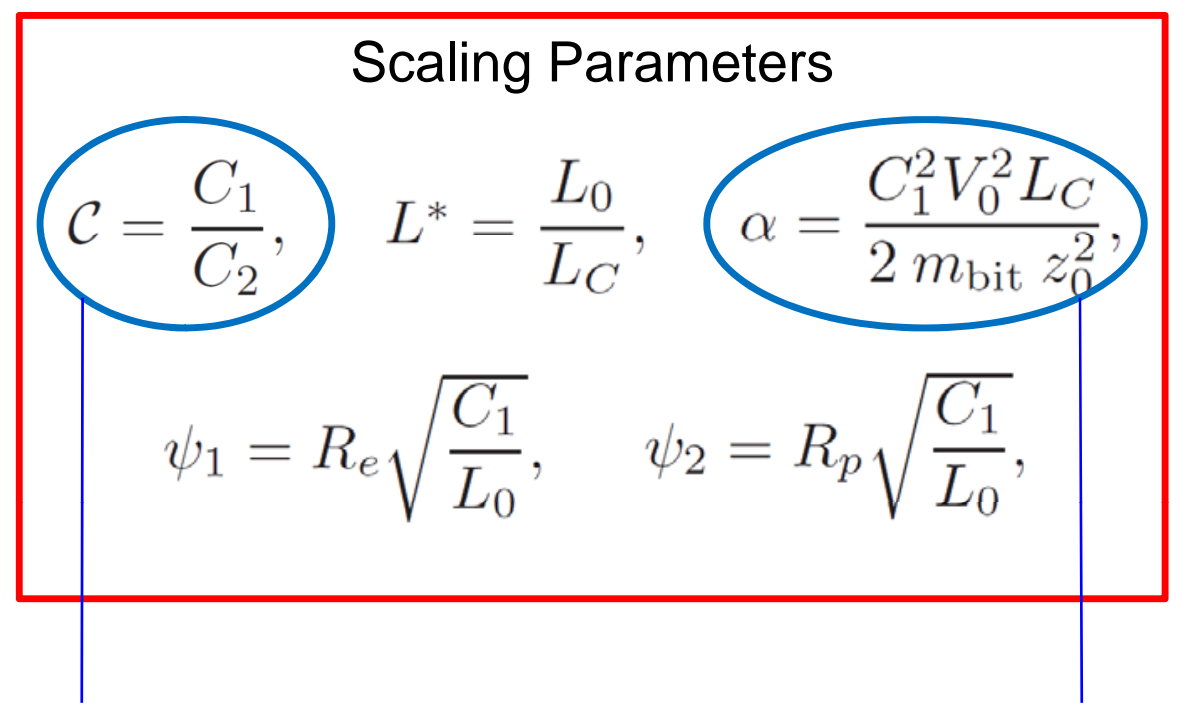

Capacitance Ratio

Dynamic Impedance 


\section{Results}

Increasing $e$ decreases swing in $I_{1}{ }^{*}$, increases ringing frequency

$$
\downarrow
$$

A)

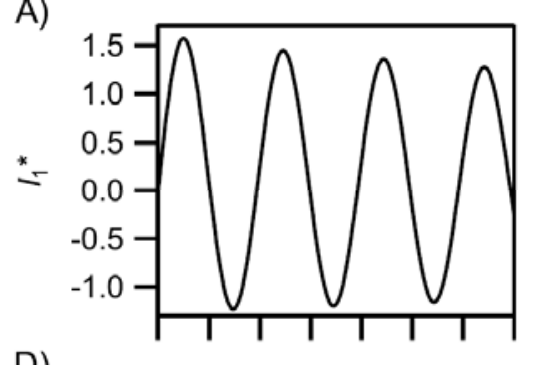

D)

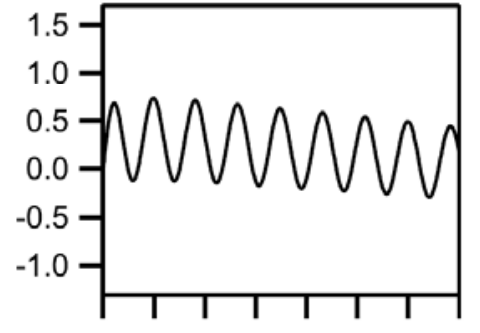

G)

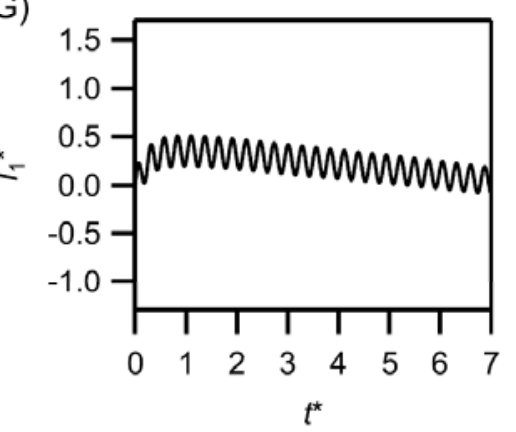

B)

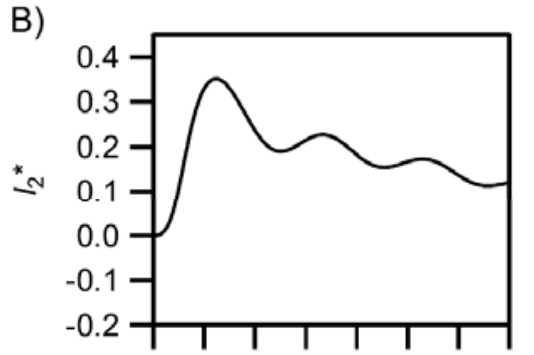

E)
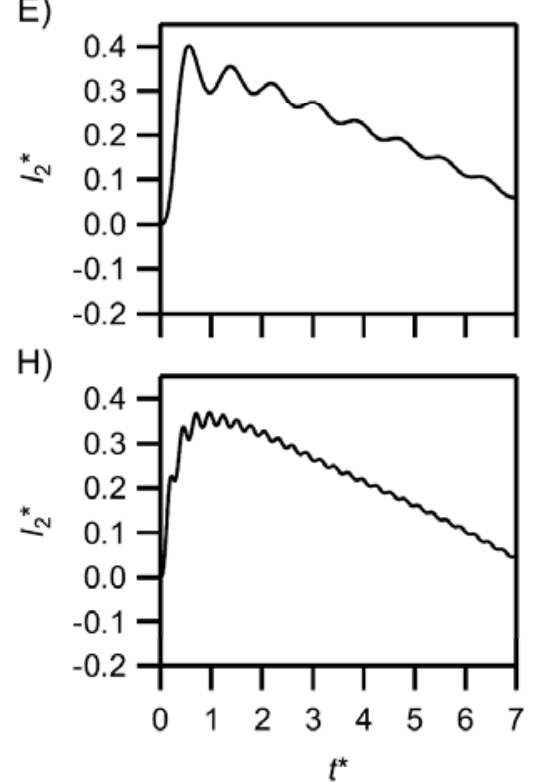

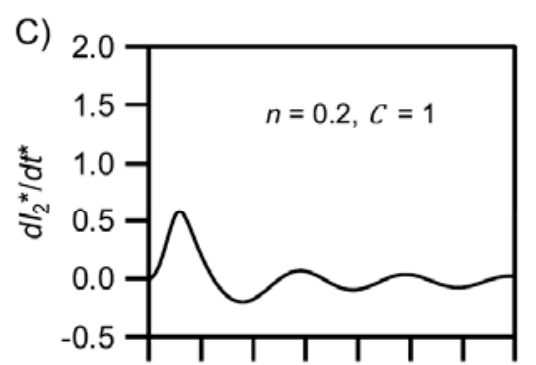

F) 2.0
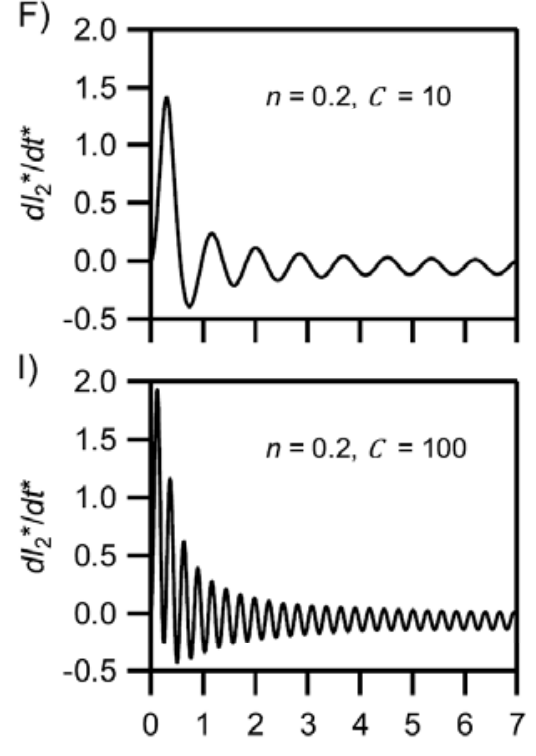

Increasing $e$ increases $d l^{\star} / d t^{\star}$ across the coil

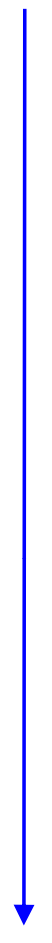

Increasing $n$ decreases amplitude and ringing frequency of $I_{1}{ }^{*}$ and $d l_{2}{ }^{*} / d t^{\star}$, but does not affect the average waveforms 


\section{Performance as a function of $\mathcal{C}$ and $\alpha$}

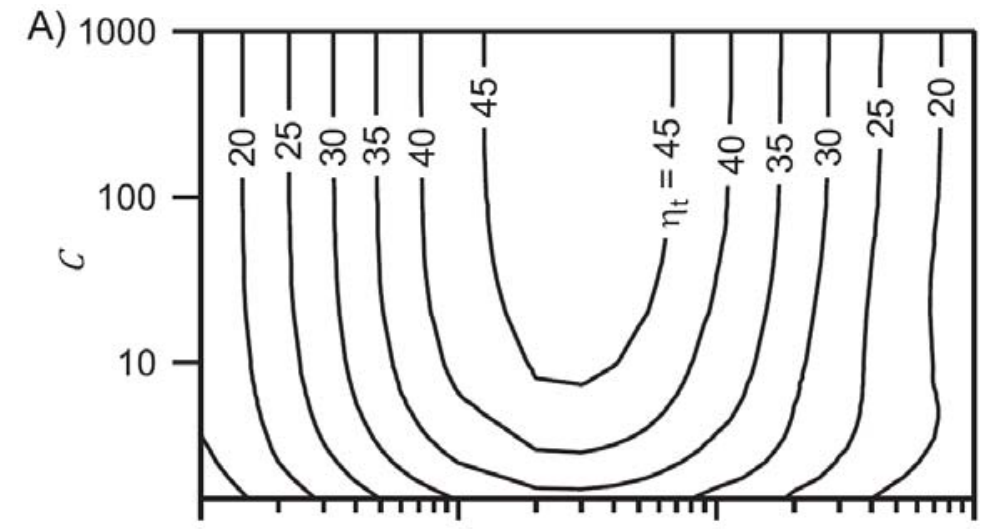

B) 1000

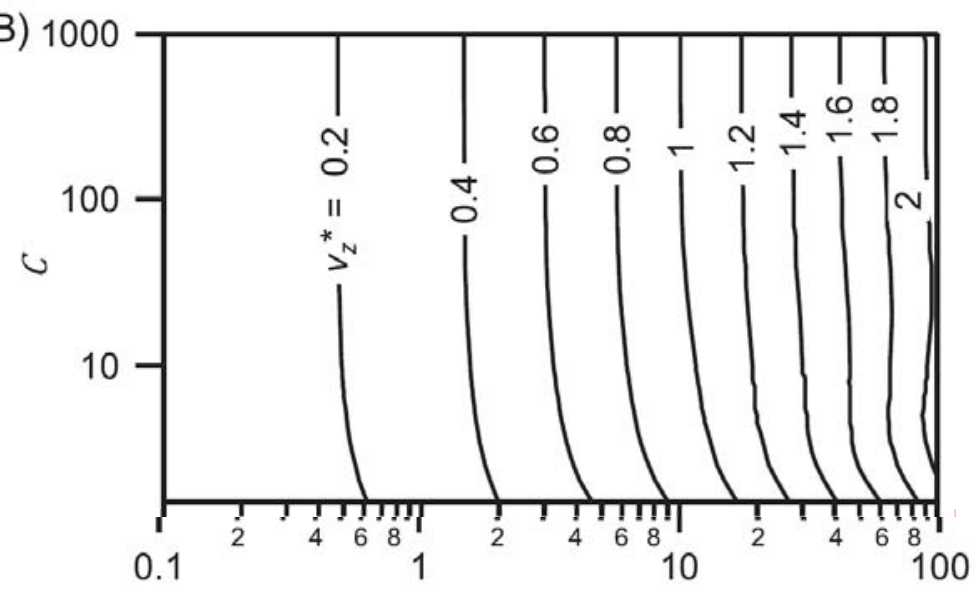

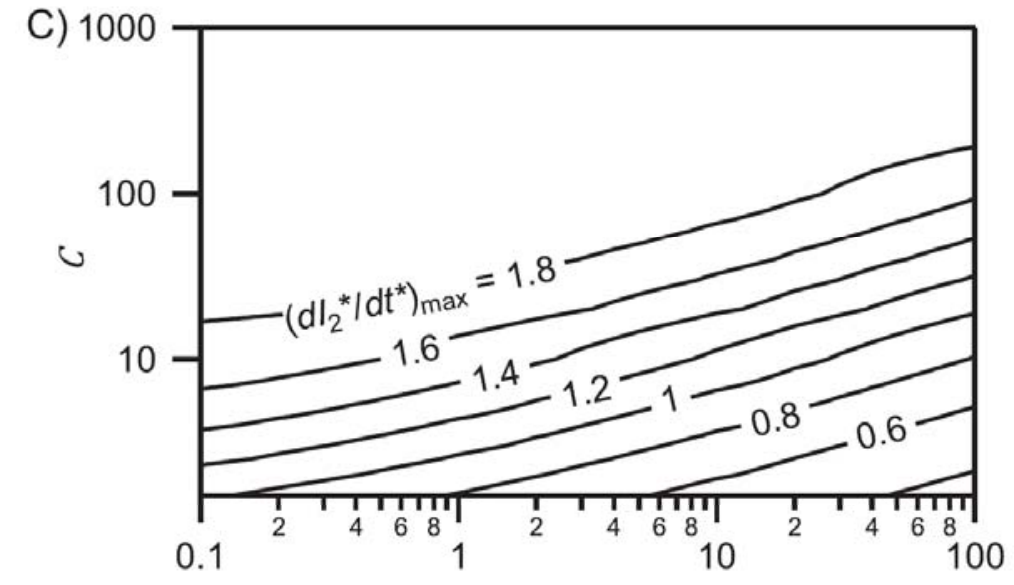

$\alpha$

- $\quad$ For fixed $\mathcal{C}$, efficiency has a maximum as a function of $\alpha$

- $\quad$ Efficiency at a constant $\alpha$ increases with increasing $\mathcal{C}$, asymptoting for $\mathcal{C}$ between 10 and 100

- $\quad$ Current rise rate increases with increasing $\mathcal{C}$, asymptoting to a value of 2 


\section{Comparing performance to single-capacitor case}
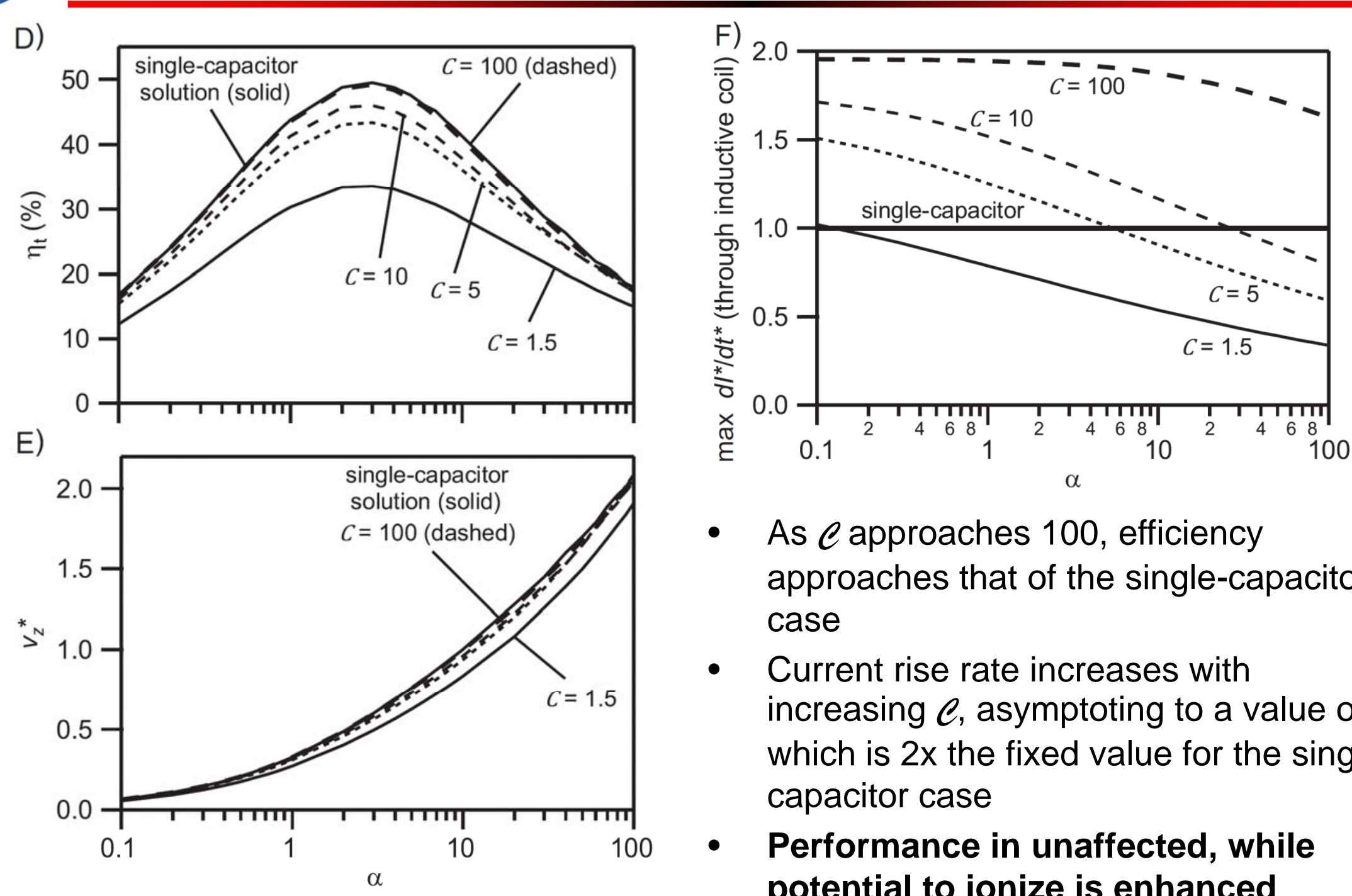

- As e approaches 100, efficiency approaches that of the single-capacitor case

- Current rise rate increases with increasing $C$, asymptoting to a value of 2 , which is $2 x$ the fixed value for the single capacitor case

- Performance in unaffected, while potential to ionize is enhanced 


\section{Conclusions}

- As in the single-capacitor case, there is an optimum dynamic impedance ratio $\alpha$ that maximizes performance

- Efficiency, maximum $d I_{2}{ }^{*} / d t^{*}$ suffer at lower values of $\mathcal{C}$, with charge oscillating between $C_{1}$ and $C_{2}$ instead of passing through the coil

- Efficiency of the two-capacitor system approaches the single-capacitor case for $C>>1$, with the commensurate $d l_{2}{ }^{*} / d t^{*}$ growing to twice the value of $d l^{\star} / d t^{\star}$ through the coil in the single-capacitor case

- As $C$ increases, the gross behavior of $I_{1}{ }^{*}$ and $I_{2}{ }^{*}$ match. The differences consist of differing high-frequency modulations overlaid upon the gross waveforms. 


\section{Acknowledgements}

- We gratefully acknowledge several helpful conversations with Mr. J. Boise Pearson during the preparation of this work

- We appreciate and acknowledge the continued MSFC support of Mr. James Martin and Ms. Mary Beth Koelbl.

- Joseph Balla's efforts on this work were supported under by the Ohio Space Grant Consortium

- This work was supported by NASA's Advanced In-Space Propulsion program managed by Dr. Michael LaPointe. 